\title{
La «médecine translationnelle» au symposium de l'ASOM
}

\section{Roxane Kübler ${ }^{a}$, Esther Kraft ${ }^{b}$, Christoph Bosshard ${ }^{\mathrm{c}}$}

${ }^{a}$ MA, responsable opérationnelle de l'Académie suisse pour la qualité en médecine (ASQM) de la FMH; ' ${ }^{b}$ lic. rer. oec., cheffe de la division Données, démographie et qualité (DDQ) de la FMH; ${ }^{c} \mathrm{Dr}$ méd., vice-président de la FMH, responsable du département Données, démographie et qualité

Lors du symposium qui se tiendra le 17 avril 2018, l'ASQM récompensera les projets qualité éprouvés du domaine de la santé avec son nouveau prix «Innovation Qualité». Cette nouvelle récompense sera remise dans le cadre d'une rencontre consacrée à un thème novateur: la "médecine translationnelle». Soyez aussi sur place et inscrivez-vous pour le symposium.

De quoi ont besoin les patients pour leur santé? Et de quoi ont besoin les professionnels de la santé pour améliorer leur travail? L'Académie suisse pour la qualité en médecine (ASQM) se penchera sur ces questions lors de son premier symposium et remettra le prix «Innovation Qualité» de l'ASQM aux pionniers en matière de qualité des soins. Cette rencontre aura lieu à Berne, l'après-midi du mardi 17 avril 2018. Le symposium mise sur l'interprofessionnalité et s'adresse aux médecins de toutes les disciplines et aux autres groupes de professionnels de santé intéressés par les questions de qualité. Il s'adresse également au public, aux patients, aux milieux politiques et aux médias. Tous y sont les bienvenus.

\section{L'innovation du laboratoire au patient en passant par la science}

En accueillant un orateur, le symposium de l'Innovation Qualité se consacrera à la question de la «médecine translationnelle» que la Société européenne de médecine translationnelle (EUSTM) définit comme «une branche interdisciplinaire de la biomédecine reposant sur trois piliers principaux: la recherche fondamentale, le traitement au chevet du patient et la communauté scientifique. L'objectif de la médecine translationnelle est de réunir les différentes disciplines, ressources, expertises et compétences techniques sollicitées pour la recherche

\section{$\left\{\mathrm{FMH}_{A S Q \mathrm{QM}}^{\mathrm{SAOM}}\right.$}

\section{Inscrivez votre projet d'ici le 8 décembre 2017}

Les projets novateurs en faveur de la qualité des soins méritent une distinction: remportez le nouveau prix «Innovation Qualité» dédié à la qualité en médecine en inscrivant votre projet sur le site www.innovationqualite.ch.

\section{Innovation Qualité: le prix destiné aux pionniers de la qualité}

Sous le nom d'Innovation Qualité, l'Académie suisse pour la qualité en médecine (ASQM) de la FMH lance un nouveau prix visant à valoriser des projets qualité éprouvés et à donner des impulsions novatrices au débat entre spécialistes. Le prix est décerné dans trois catégories, dont deux dotées de 15000 francs chacune, Repenser la prise en charge des patients et Sécurité des patients, et une dotée de 10000 francs, Organisations médicales. Soumettez dès à présent votre projet pour le prix Innovation Qualité! Le délai d'inscription est fixé au 8 décembre 2017. Vous trouverez de plus amples informations sous www.innovationqualite.ch. 
Correspondance:

Fédération des médecins suisses, FMH

Division Données,

démographie et qualité

Elfenstrasse 18

Case postale 300

CH-3000 Berne 15

roxane.kuebler[at]fmh.ch

Tél. 0313591111 fondamentale (le laboratoire), le traitement auprès du patient et le monde des sciences, respectivement des scientifiques, afin de promouvoir l'évolution et l'amélioration de la prévention, du diagnostic et des traitements.» [1, 2] En d'autres termes, les nouvelles découvertes de la recherche et du développement doivent sortir de leur cocon. Elles doivent permettre de donner naissance à des produits et des instruments novateurs, diffusés ensuite sur le marché pour finalement atteindre les patients.

\section{La médecine translationnelle en théorie et en pratique}

L'Institut suisse pour la médecine translationnelle et l'entrepreneuriat (sitem-insel) vise à construire, exploiter et développer davantage un centre de compé- tences national à Berne [3]. Le Prof. Dr, em. Felix Frey, directeur de sitem-insel, invité au symposium, présentera les chances et les bénéfices pour les patients.

\section{Inscription}

Vous pouvez vous inscrire dès maintenant pour le symposium de l'ASQM: innovationqualite.ch $\rightarrow$ Remise des prix au symposium (de l'ASQM). Nous vous attendons.

\section{Références}

1 Randall J. Cohrs, Tyler Martin, Parviz Ghahramani, Luc Bidaut, Paul J. Higgins et Aamir Shahzad: Translational Medicine definition by the European Society for Translational Medicine, New Horizons in Translational Medicine, mars 2015, volume 2 (3), p. 86-88. http:// www.newhorizonsintranslationalmedicine.com/article/s23075023(14)00078-2/pdf [4.10.2017].

2 https://de.wikipedia.org/wiki/Translationale_Medizin [4.10.2017].

3 cf. http://www.sitem-insel.ch [4.10.2017]. 